\title{
Smart Cities - How can we get bridges talking?
}

\section{Paul Anthony Cahill}

School of Engineering - Civil \& Environmental Engineering. , UCC

The story of civilization is, in a sense, the story of engineering - that long and arduous struggle to make the forces of nature work for man's good. (L. Sprague de Camp)

\section{Technology - evolving but not universally}

With the abundance of smart technology that has been developed in recent times, society is ever progressing to improve the comfort, quality and safety of our lives and the world around us. Such technological developments have resulted in great improvements in a variety of fields and have greatly enhanced our communications, leisure and health. However, much of the most fundamental infrastructure that we rely on most for our everyday needs has yet to benefit from the information technology revolution, namely civil infrastructure. From the water we use first thing in the morning to shower and brush our teeth, to the roads and bridges we use to travel, civil infrastructure is vital to our everyday lives. Not only that, but improved efficiency in all these areas is required to help protect the environment for future generations and reverse the excess that has damaged our planet in the past two centuries.

\section{How smart are smart cities?}

There has been intense focus in recent years on the development of smart cities, which act as a system with technology being integrated into all aspects of life. Amongst a host of other engineering infrastructure, such as power, transport around such cities has received great benefits from these developments. It is only in the customer interface that benefits of such technology have been seen with real time traffic and public timetables information being freely available, whilst the actual infrastructure, such as bridges that sustains this transport system, still remains lacking in many aspects. The health of such bridges are still determined primarily through visual inspections, which can only indicate surface degradation of the bridge and are dependent on the experience and competence of the inspector. The other options currently available include wired sensing, which requires untenable levels of man hours and disruption to the service, destructive testing, which involves damaging the structure to determine its health before repairing the imposed damage, or wireless sensors, which rely on batteries that have short lifespans and need frequently to be changed. The ongoing development of smart technology raises the 
question, how can we integrate such technology with bridges? How can we improve our knowledge of the health of bridges, which will help prioritise maintenance for improved safety and economics? And, finally, can we get bridges talking and truly develop smart cities?

\section{Bridges as a source of energy}

My research focuses on the integration of smart energy harvesting sensors with civil infrastructure, with emphasis on bridge structures. These sensors are designed around active piezoelectric materials, which have the ability to covert changes in strain energy into electrical energy through the direct piezoelectric effect. What this means is that when attached to a host structure, the sensor will convert any movements in the host into electrical energy and so has the potential to create a wireless sensor node which is independent of external power supplies, such as batteries. There have been intense investigations into the development of such energy harvesting devices but this has primarily focused on when the sensors undergo stable simple harmonic motion, whereby the hosts' movement has a constant frequency and amplitude. In reality, the vast major of civil infrastructure do not experience such motion but instead experience live loadings, which are best described as unstable loading, such as the wind or vehicles passing over a bridge. It is therefore imperative to investigate how the sensors will interact with structures under real world conditions to fully realise their potential.

One such interaction that is very appealing is the development of such sensors for train bridges (Figure 1). This is due to trains having such a large load and also the timetabled nature of the rail network allows for a highly predictable level of loading on the bridge. This means that the energy harvesting sensors will have not only a high level of electrical energy output but also be highly predictable in the amount of energy it can harvest in a given time period. Train bridges are therefore the perfect host structure with which to integrate smart energy harvesting sensors. The challenge therefore is the design of the energy harvesting sensors which can capture the live loading of the train passage and accurately calibrate the device for other applications such as damage detection and alerts for the control of vibrations in a structure.

\section{A close-up view of the energy harvesting sensors}

There are two device configurations that I am investigating for the creation of the energy harvesting sensor. The first type of device is a patch based solution, where the energy harvesting material is attached directly to the bridge by using a bonding agent. In this way, electrical energy is generated by the harvester due to the changes in the strain energy on the surface of the bridge as a vehicle passes over it. The second type of device is a cantilever based harvester. The cantilever based harvester is excited by the acceleration 


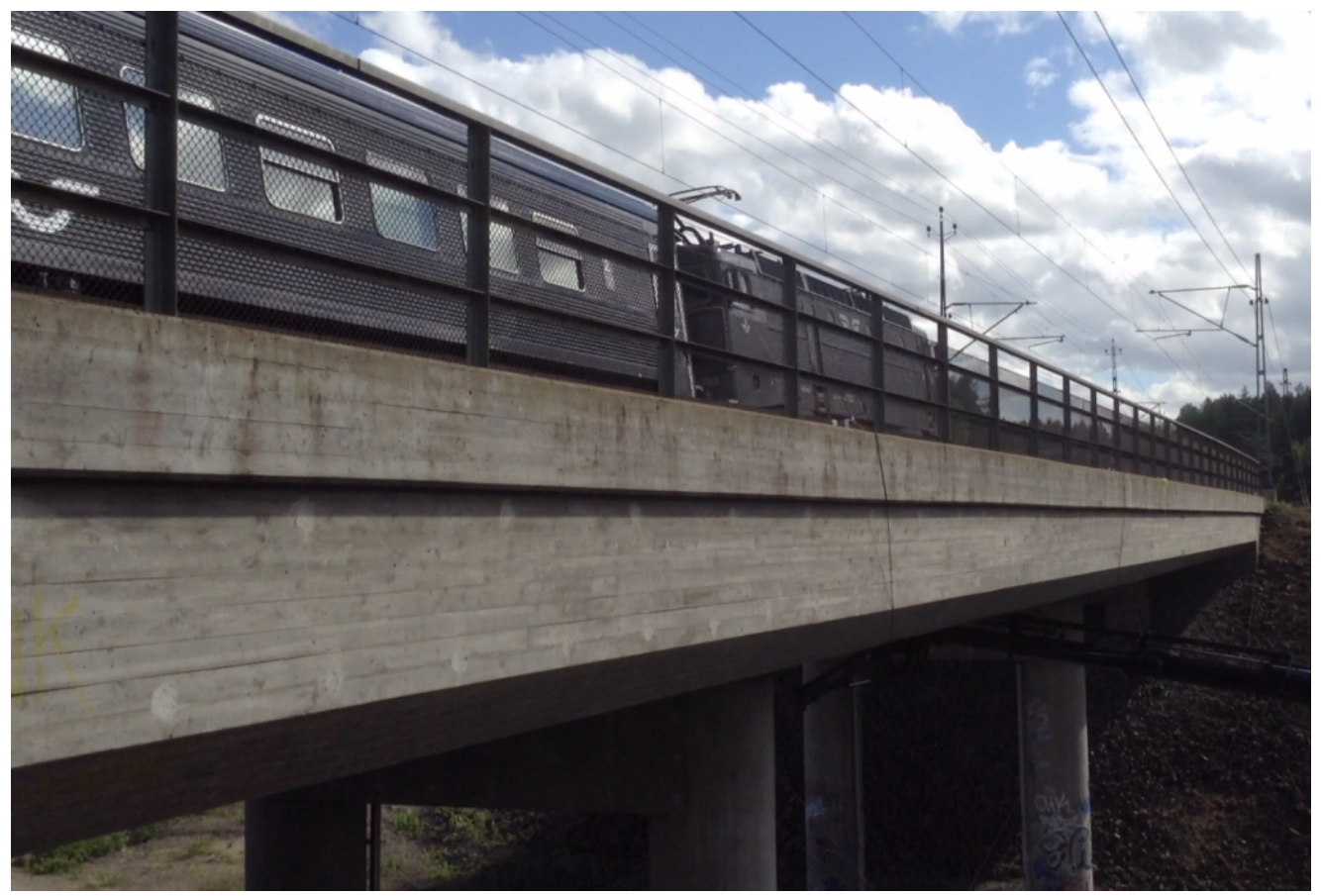

Figure 1: Example of Train Passage over Bridge. Source: Author

of the bridge and transfers this vibration energy to its substrate which is then in turn converted into electrical energy by the piezoelectric material. The cantilever device is attached by its base to the host structure and has a tip mass at the free end (Figure 2). This tip mass can be changed so that the natural frequency of the harvester can be matched to that of the bridge, this is an optimisation of the harvesting device.

As previously mentioned, to maximise the energy harvesting output of the sensor, it must be designed in such a way so as to match the natural frequency of the bridge or the frequency at which the maximum amplitude of vibration occurs. Most often these two

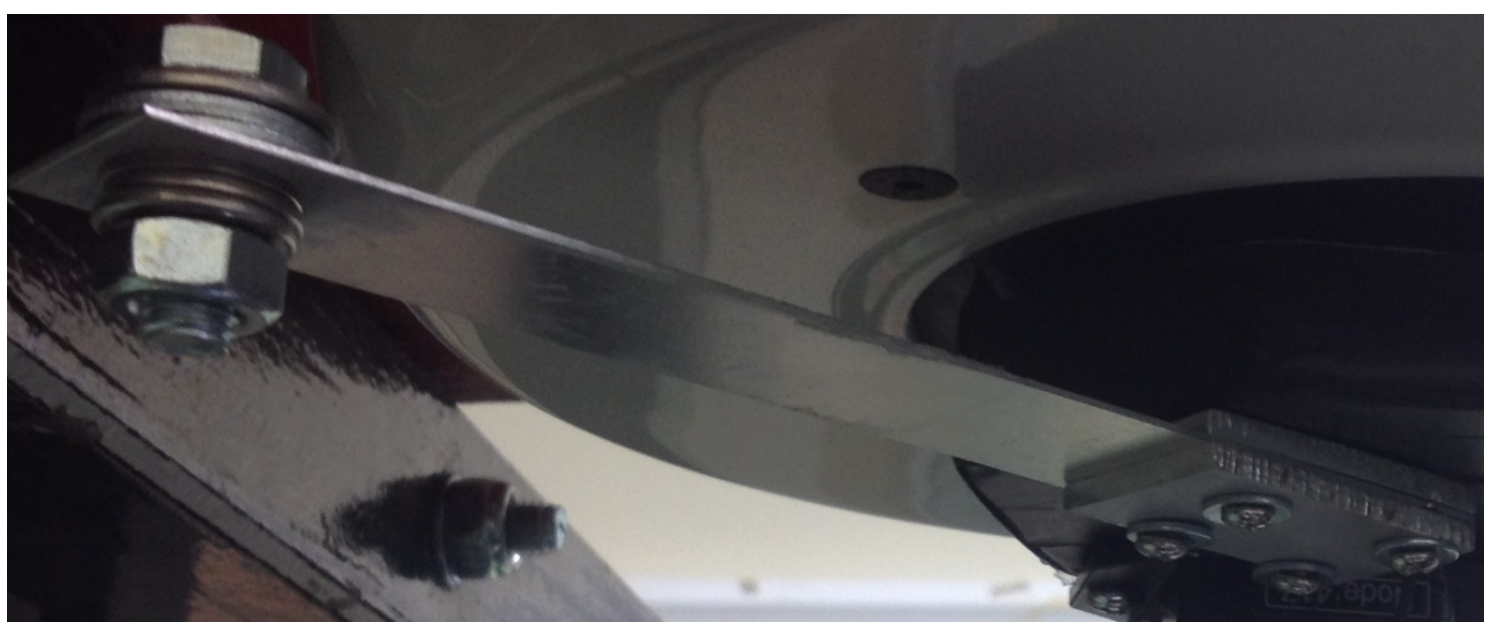

Figure 2: Cantilever Device for Energy Harvesting from Train Induced Vibrations in Bridges. Source: Author 


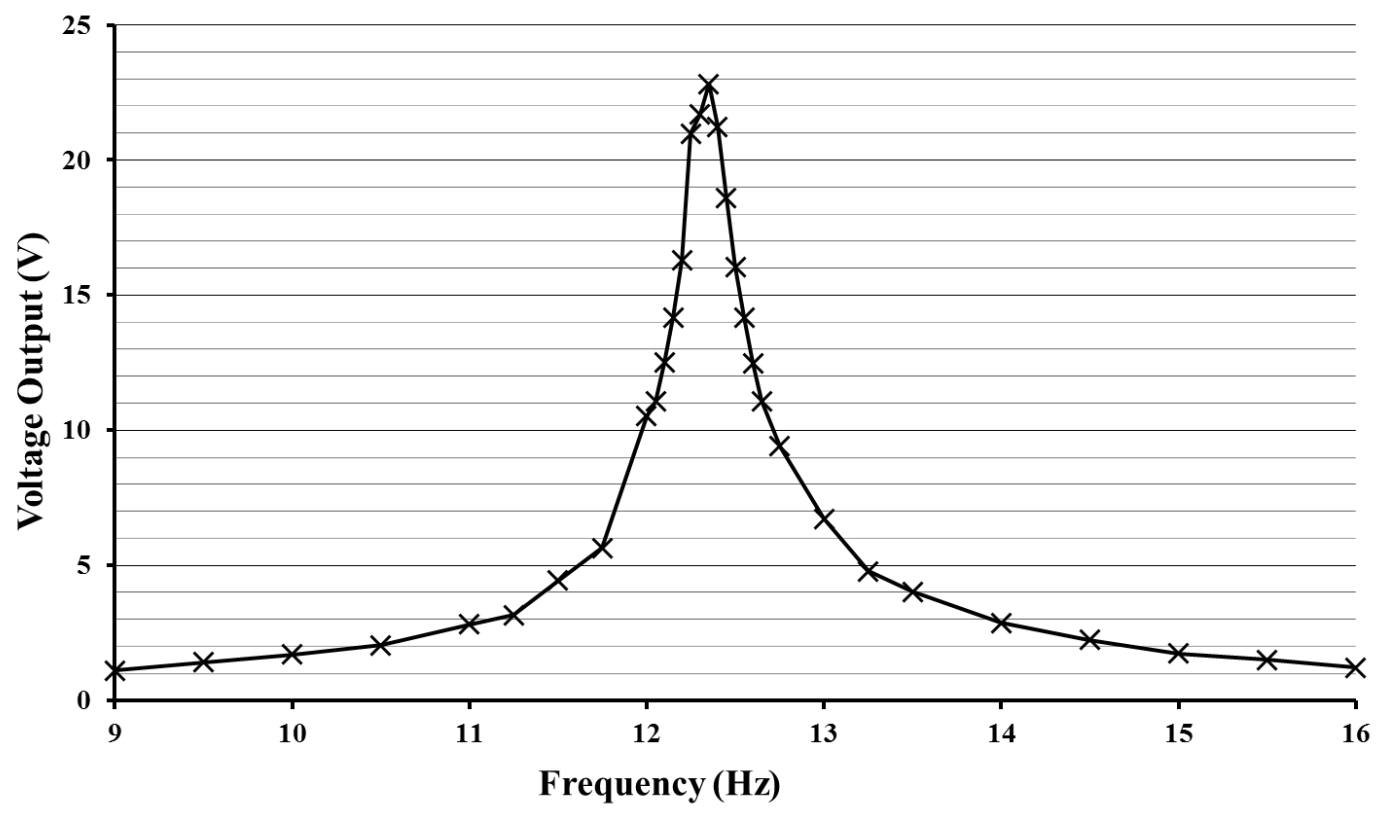

Figure 3: Example Voltage Response versus Frequency of a Cantilever Device Tuned to 12.6Hz. Source: Author

frequencies are the same however they may be different depending on the type of bridge being considered. The cantilever device returns a maximum voltage output at a single frequency, which means as we move away from that frequency the amount of electrical energy that is generated drops sharply (Figure 3). Therefore, it imperative that the natural frequency of the device is tuned correctly to that of the host structure, so as to maximise the potential energy that can be harvested.

The amount of electrical energy that can be harvested from a single train passage is quite low, in the range of milliwatt, however with the addition of a storage circuit and battery, the energy which is harvested from each train passage can stored. This can then be used to power a wireless node which can transmit data such as environmental information or, more appealing, the health of a bridge to a centralised computer. If all bridges were to be fitted with such energy harvesting sensors, the health of our cities bridges could be monitored using a single computer.

\section{A health check-up for bridges}

It is often the case that the only time that we hear about damage in bridges is when the worst case happens and sudden, catastrophic collapse occurs. However, damage can occur in many different forms and can attack bridges in many different ways, from the most visible, such as vehicles striking the bridge and cracks developing (Figure 4), ), to the more concealed, whereby the internal sections of the bridge suffer damage such as corrosion. Energy harvesting sensors can act as a monitor for such damage and can enable authorities to prioritise maintenance or alert to the need for immediate action. This can be done in 


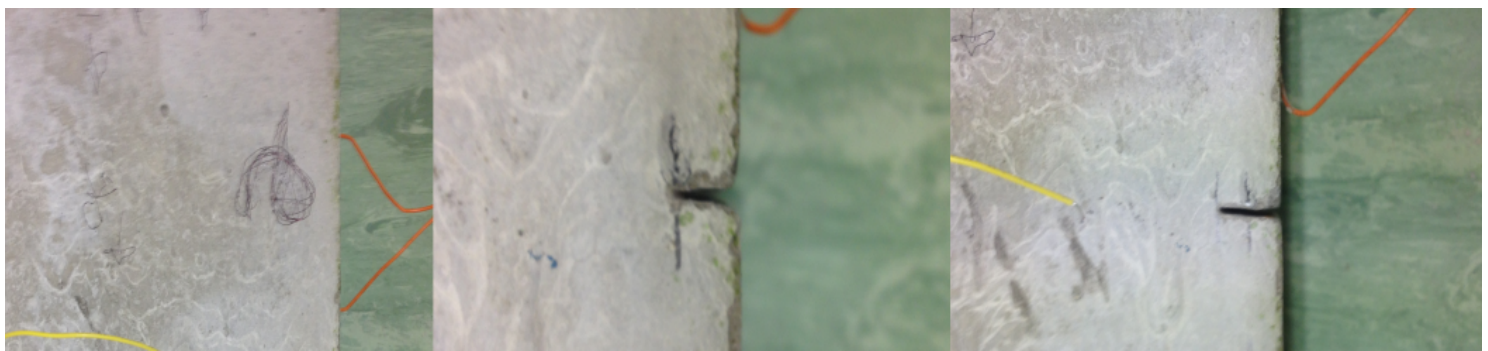

Figure 4: Evolution of Damage Crack in a Reinforced Concrete Beam. Source: Author

two ways. First is to use the voltage signal directly and convert from the time to frequency domain. As damage affects a bridge, the natural frequency of the structure changes and as it does so, the voltage output will be influence, which will therefore make it possible to track the evolution of the damage within the host bridge. The second way of detecting damage is to use the amount of energy harvested by the device. As damage affects the bridge, the acceleration and strain variations which provide the necessary means for the device to harvest energy will change. The amount of energy which is harvested will change and thus indicate the presence of damage. Through the use of an array of such devices, the location and magnitude of the damage can also be ascertained, which will provide invaluable information for bridge maintenance authorities and connect the bridge to the smart cities.

\section{Bringing the bridge to the lab}

Full scale testing of train bridge interaction is costly in terms of economics and man hours. It is therefore much more attractive to determine the performance of the energy harvesting device and its applications in a laboratory based setting first. As part of my research, I have developed a procedure that will achieve this objective (Figure 5). The procedure takes into account the type of device which requires validation and the type of data which is available. Should both strain and acceleration data be available, from either experimental or theoretical studies, both types of devices can be validated. The performance of the cantilever device can be determined by using a Permanent Magnet Shaker, which can replicate any given accelerations in a laboratory setting, and applying the same accelerations as that which are caused by train - bridge interaction. This means that the scale of the applied acceleration experienced by the device would be the same in both cases and thus the cantilever devices performance would be equal in the lab as it would be attached to the bridge. Similarly for the patch device, through the use of the procedure but this time using a fatigue testing device which can apply the same strains as that of the train bridge interaction, the output of the patch device can be determined. The application of damage detection can also be verified in a laboratory setting through this procedure as the strains and accelerations of the damaged and undamaged cases for the bridge can be replicated and the performance of the bridge for both determined. 


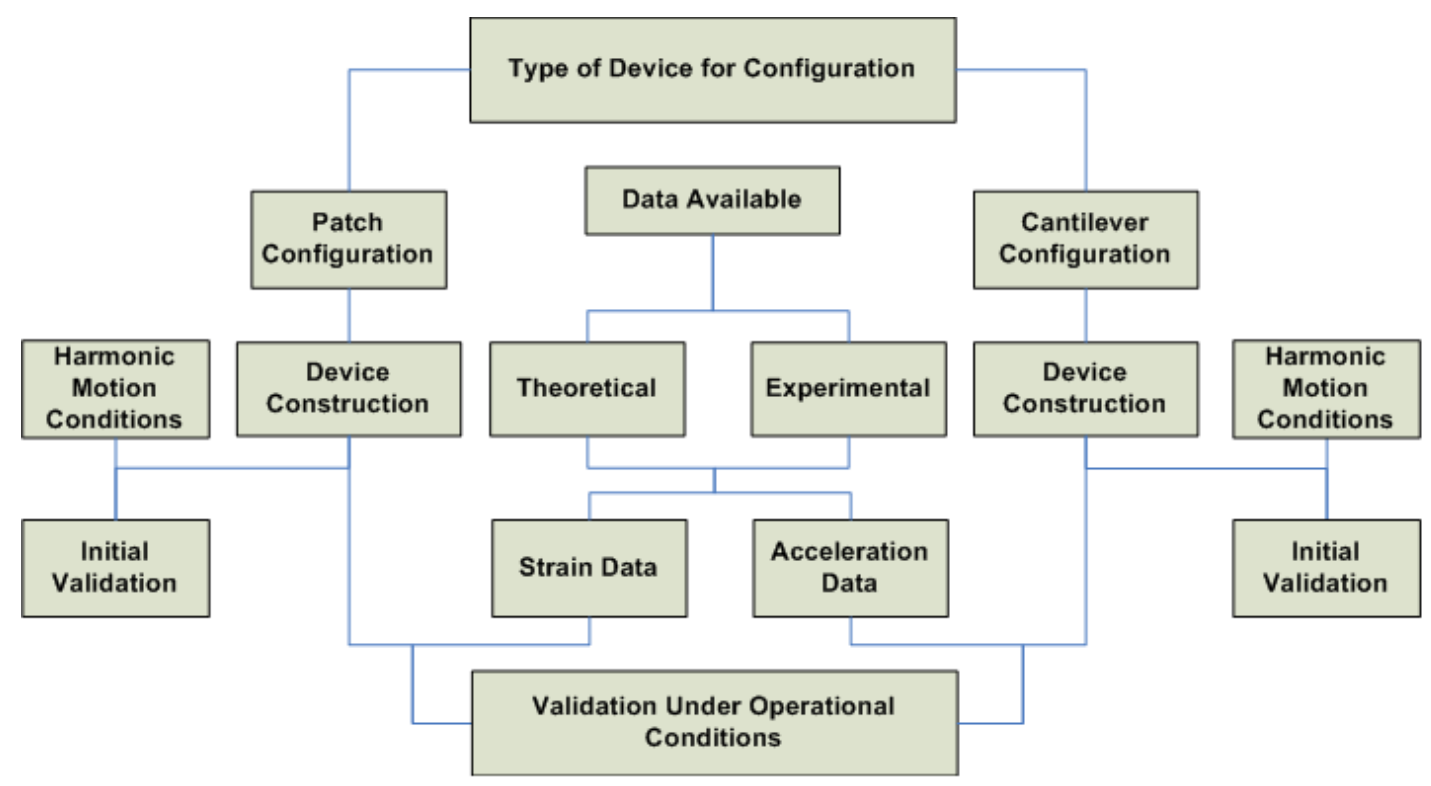

Figure 5: Procedural Flow Chart for Validation of Energy Harvesting Sensors for TrainBridge Interaction. Source: Author.

Source: Author

\section{Self-powered wireless sensors - A voice for bridges}

Through the use of my proposed energy harvesting sensors, coupled with an AC - DC storage circuit and a wireless node, wireless sensors can be developed for bridge applications. The health of the bridges will be determined in a very inexpensive way and the information on maintenance requirements will be greatly enhanced. These smart energy harvesting sensors will allow for bridges to finally gain a voice in the smart cities around us.

The author is a student in the Dynamical Systems \& Risk Laboratory (DSRL), School of Engineering, UCC under the supervision of Dr. Vikram Pakrashi and Dr. Alan Mathewson. The author would like to thank Dr. Nathan Jackson for his assistance with this research. This research was partly carried out at Tyndall National Institute. 